\title{
The Attraction of Water for Itself at Hydrophobic Quartz Interfaces
}

DOI:

10.1021/acs.jpcb.0c04545

\section{Document Version}

Accepted author manuscript

Link to publication record in Manchester Research Explorer

\section{Citation for published version (APA):}

Henchman, R. (2020). The Attraction of Water for Itself at Hydrophobic Quartz Interfaces. The Journal of Physical Chemistry B, 124(29), 6369-6375. https://doi.org/10.1021/acs.jpcb.0c04545

\section{Published in:}

The Journal of Physical Chemistry B

\section{Citing this paper}

Please note that where the full-text provided on Manchester Research Explorer is the Author Accepted Manuscript or Proof version this may differ from the final Published version. If citing, it is advised that you check and use the publisher's definitive version.

\section{General rights}

Copyright and moral rights for the publications made accessible in the Research Explorer are retained by the authors and/or other copyright owners and it is a condition of accessing publications that users recognise and abide by the legal requirements associated with these rights.

\section{Takedown policy}

If you believe that this document breaches copyright please refer to the University of Manchester's Takedown Procedures [http://man.ac.uk/04Y6Bo] or contact uml.scholarlycommunications@manchester.ac.uk providing relevant details, so we can investigate your claim.

\section{OPEN ACCESS}




\section{The Attraction of Water for Itself at Hydrophobic Quartz Interfaces}

Quinn A. Besford*, ${ }^{\dagger}$ Andrew J. Christofferson*, $\ddagger$ Jas Kalayan, Jens-Uwe Sommer, " and Richard H. Henchman $₫$

$\dagger$ Institute of Physical Chemistry and Polymer Physics, Leibniz Institute for Polymer Research Dresden e.V., Hohe Str. 6, 01069 Dresden, Germany $\ddagger$ School of Science, RMIT University, Victoria 3001, Australia

\Manchester Institute of Biotechnology, The University of Manchester, Manchester, United Kingdom

§School of Chemistry, The University of Manchester, Oxford, United Kingdom

|| Institute Theory of Polymers, Leibniz Institute for Polymer Research Dresden e.V., Hohe Str. 6, 01069 Dresden, Germany 


\begin{abstract}
Structural forces within aqueous water at a solid interface can significantly change surface reactivity and the affinity of solutes towards it. We show by molecular dynamics simulation how hydrophilic and hydrophobic quartz surfaces perturb the orientational structure of aqueous water, ultimately strengthening dipolar forces between molecules in proximity to the interface. When derived as a function of distance from each surface, it was found that both surfaces indirectly enhance the long-range dipolar attraction of water for itself towards the interfacial region. This was found to be longer-ranged for water molecules solvating the hydrophobic surface than those solvating the hydrophilic surface, with a range of up to $2.5 \mathrm{~nm}$ from the hydrophobic surface. Our results give direct quantification of surface-induced changes in solvent-solvent attraction, ultimately providing a counterintuitive addition to the balance of hydrophobic forces at aqueoussolid interfaces.
\end{abstract}




\section{Introduction}

Intermolecular forces within aqueous water at solid interfaces are complex, and implicated in biological, geological, and chemical phenomena (e.g. surface adhesion of proteins and cells, ${ }^{1}$ geosequestration of carbon dioxide, $\stackrel{[2]}{2}$ and aqueous phase heterogenous catalysis, ${ }^{[-3}$ respectively). The short length scales, presence of impurities (e.g. dissolved gas), and difficulty in preparing clean, extended surfaces mean that they are also tantalizingly difficult to measure. ${ }^{4-7}$ In the interfacial region, a combination of non-bulk-like molecular orientation,, 8

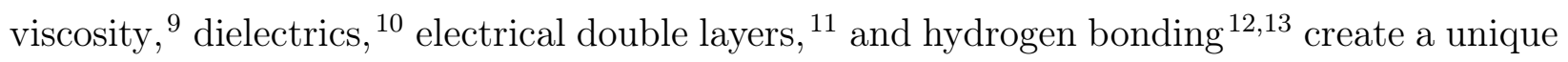
solvation environment that can significantly change the properties of the surface itself. This leads to a highly dynamic region within the adjacent solvating liquid that can exert forces on ions, molecules, and macroscopic objects in close proximity to the surface. $\frac{14}{17}$ The length scales pose challenges to both experimental and theoretical investigations, where measurements of the narrow interfacial region are complicated by the requirement to enhance the signal from the interface over those of the adjacent bulk liquid and solid phases. $\frac{18}{18}$

Information on the structure and dynamics at solid-aqueous interfaces may be obtained by sum-frequency generation (SFG) spectroscopy, 1921 which in combination with molecular dynamics $(\mathrm{MD})^{[22}$ provides details on the structural and density perturbations within aqueous water. Relating these molecular orientations and density oscillations to the intermolecular forces towards the interface (i.e., what forces "act" on solutes towards the interface) is not so straightforward. Forces towards solid-aqueous interfaces are typically probed by measurement of the forces between two surfaces immersed in aqueous water via atomic force microscopy (AFM) or surface force apparatus (SFA) measurements. The forces are either attractive or repulsive, strong or weak, and dependent on ionic strength and surface type.23|24 They also typically exceed estimated van der Waals (vdW) forces between the surfaces across the water medium, leading to the notion of a so-called "hydrophobic force". 25 This force has been attributed to various causes, $\stackrel{4}{4}$ including charge correlations between patchy bilayers on each surface ${ }^{26}$ and capillary bridging bubbles. ${ }^{[27}$ However, theoretical treatments 


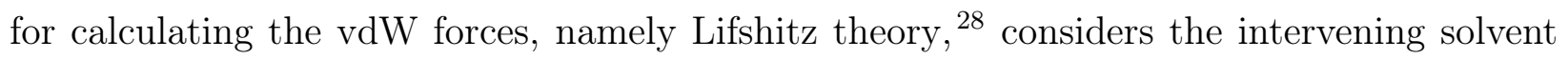
to be a structureless dielectric continuum, thereby neglecting the effect of intervening liquid structure on the forces between the surfaces. Establishing a concrete link between the interfacial water molecular orientation and resulting forces will therefore allow more insight into solvent-mediated forces towards and between surfaces in aqueous solution.

Herein, using MD simulations we probed the perturbation of aqueous water structure in close proximity to model hydrophobic and hydrophilic quartz interfaces. Our interest in quartz-aqueous water interfaces stemmed from their ubiquitousness in nature and recent

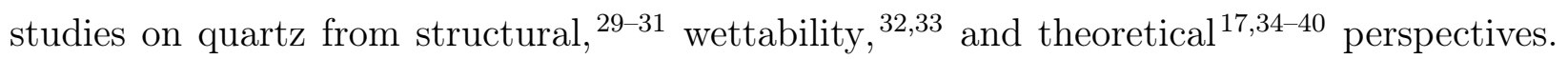
The dipolar potential of mean force between water molecules as a function of distance from each surface was calculated, revealing a new component of the solvent free energy towards the interfacial region. Unexpectedly, whilst this component was steeper (greater free energy gain) towards the hydrophilic surface, it was longer ranged towards the hydrophobic surface, where stabilizing forces extended up to approximately $2.5 \mathrm{~nm}$ from the hydrophobic surface. Our results highlight a solvation mechanism that is neglected in continuum theories of vdW forces in aqueous water towards solid surfaces, and that is the surface-induced structural enhancement in water-water interactions.

\section{Methods}

Simulations were performed using the published parameters of the consistent valence force field $(\mathrm{CVFF})$ for the quartz surfaces. $\frac{41}{4}$ The SPC/E model was used for water, $\frac{42}{4}$ and the Kirkwood-Buff parameters for $\mathrm{Na}^{+}$and $\mathrm{Cl}^{-} .43$ The open-source Large-Scale Atomic/Molecular Massive Parallel Simulator (LAMMPS) MD code ${ }^{44}$ was used for all simulations. Non-bonded interactions were cut-off at $12 \AA$, with a switching function applied between 10 and $12 \AA$. Electrostatics beyond the cut-off were evaluated with the particle-particle-particle-mesh (PPPM) summation method ${ }^{45}$ with an accuracy of $10^{-5} \mathrm{kcal} \mathrm{mol}^{-1}$. Surface simulations were run 
in the NVT ensemble, while the bulk aqueous water was run in the NPT ensemble. A Nosé-Hoover thermostat and barostat were applied to maintain a temperature of $298 \mathrm{~K}$ and a pressure of 1 atm, with a time step of $1 \mathrm{fs}$, and an output frequency of $20 \mathrm{ps}$. All simulations were run for 25 ns, with equilibrium for the first 5 ns, and analyses performed on the final 20 ns. Atomic coordinates of quartz were frozen during simulation, except for the

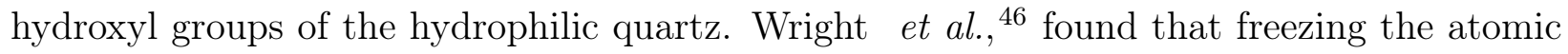
coordinates of the quartz had little effect on the water structuring.

Two $\alpha$-quartz (001) surfaces were built from an original unit cell $\underline{47}$ of dimensions 4.913 $\times 8.5096 \times 5.4052 \AA^{3}$, which was replicated by 9,5 , and 10 in the $x, y$, and $z$ dimensions, respectively, to create a slab of dimensions $44.217 \times 42.548 \times 54.052 \AA^{3}$. To construct the model hydrophobic surface, the outer layer of dangling oxygens in the positive $z$ direction was stripped, and silicon atoms at the positive and negative $z$ interfaces were protonated (Figure 1A). To construct the model hydrophilic surface, the terminal silicons of the outer layer in the negative $z$ direction were oxygenated, and oxygen atoms at the positive and negative $z$ interfaces were protonated $($ Figure $1 \mathrm{E})$. Optimal cell dimensions were determined by placing 2640 water molecules on either side of each respective quartz surface and equilibrating for 25 ns. Final cell dimensions were determined from water density profiles, where the total cell length in the $\mathrm{z}$ direction was determined from $50 \%$ of the water density on each side of the quartz, with a vacuum space of $\approx 60 \AA$ separating the water halves. Final cell dimensions were $44.217 \AA \times 42.548 \AA \times 141.500 \AA$ and $44.217 \AA \times 42.548 \AA \times 139.500 \AA$ for the hydrophobic and hydrophilic surfaces, respectively. The effect of the terminal groups on the wetting of the surfaces was determined by separately simulating a droplet of 6140 water molecules placed on surfaces extended in the $x$ and $y$ for final dimensions of 132.651 $\AA \times 127.644 \AA$, respectively. After equilibrating for $4 \mathrm{~ns}$, it was found that the hydrophobic surface maintained a well defined droplet with a contact angle of approximately $137^{\circ}$ (Figure S1), consistent with previous experimental results for quartz modified with methyltrichlorosilane $\left(136^{\circ}\right) . \stackrel{48}{1}$ On the other hand, the hydrophilic surface was completely wet by 
the droplet (Figure 1B and F, respectively). For the larger-scale analysis, both quartz systems contained 5280 water molecules with $150 \mathrm{mM} \mathrm{NaCl}$. This ionic strength was purposely chosen to keep our study relevant to biological processes, where salt ions are ubiquitous. $\frac{49}{4}$ Simulations were performed for 25 ns, with snapshots saved every 20 ps. Snapshots of the equilibrated systems are shown in Figure $1 \mathrm{C}$ and $\mathrm{G}$, and unless otherwise stated, $\mathrm{z}=0 \mathrm{~nm}$ is taken from the outermost silicon atom of each surface, respectively.

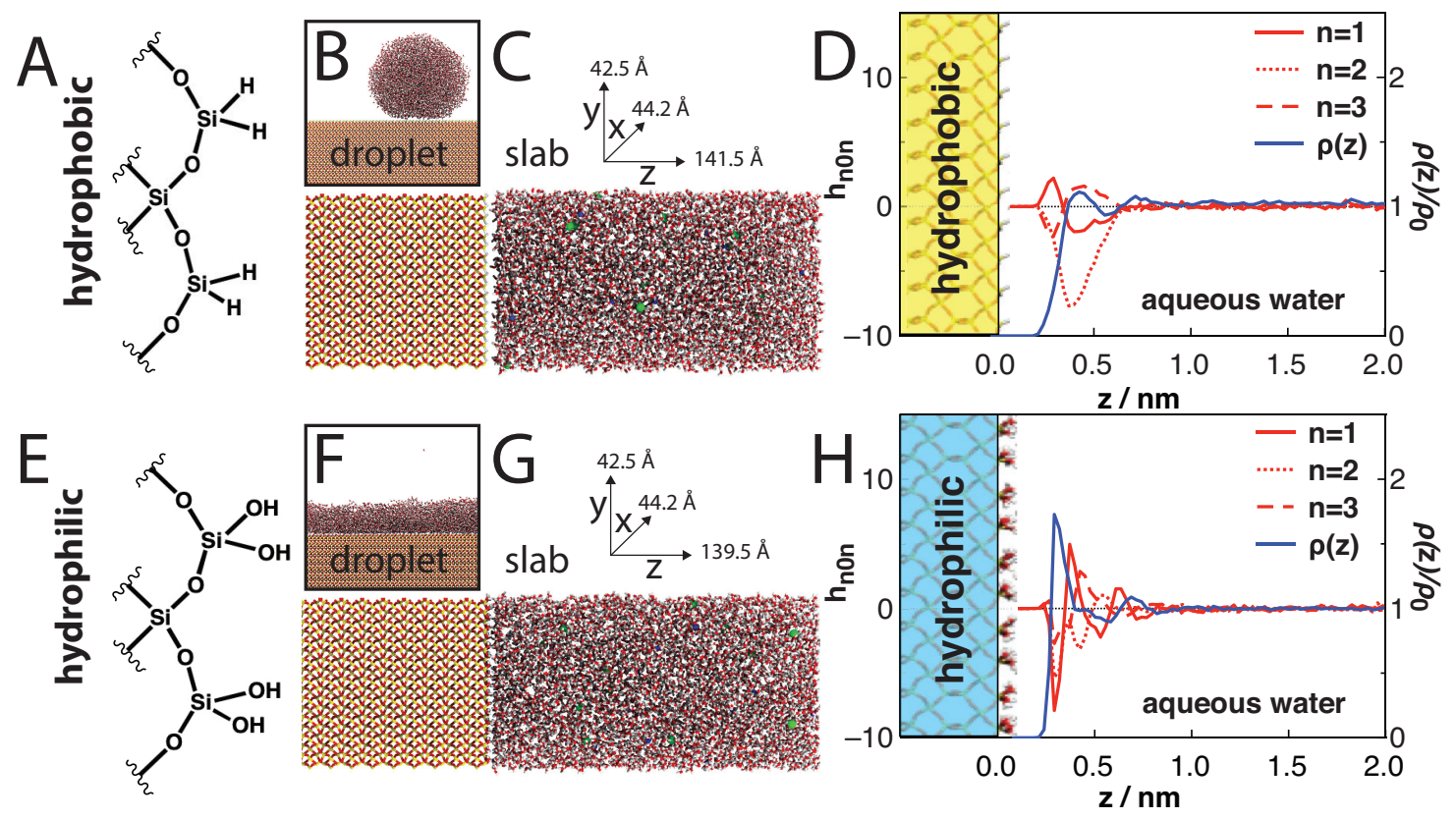

Figure 1. A schematic of the surface chemistry (A and E), snapshots of water droplets on quartz (B and F), and equilibrated slab geometry snapshots of aqueous water and hydrophobic and hydrophilic quartz $(\mathrm{C}$ and $\mathrm{D})$, respectively, along with the corresponding density and dipolar orientation profiles ( $\mathrm{D}$ and $\mathrm{H}$ ). Note the aqueous water consists of $150 \mathrm{mM} \mathrm{NaCl}$, shown as blue and green spheres, respectively.

\section{Results \& Discussion}

The molecular orientations of water molecules near the quartz surfaces were probed through specific correlation functions that describe preferential modes in dipolar orientations with respect to the interface normal, $\mathrm{h}_{n 0 n}$, along with the density profile, $\rho(z)$ Figure $1 \mathrm{D}$ and H). It was found that water adjacent to the hydrophilic surface has a higher and sharper 
density profile than the hydrophobic, reflecting an increased preference for water to solvate the hydrophilic surface. For the hydrophobic interface, there is a region of diminished water density consistent with previous reports of a "hydrophobic gap" of water at hydrophobic surfaces. .50151 The density of ions was found to be higher towards hydrophilic surface than the hydrophobic (Figure S2), along with an increased number of hydrogen bonds ${ }^{52}$ at a closer distance to the interface (including water-surface bonding), confirming water-quartz hydrogen bonding for the hydrophilic system that is absent in the hydrophobic (Figure S3). Furthermore, an extended perturbation of the hydrogen-bond distributions between water molecules away from each surface was found with respect to the number of hydrogen bond donors and acceptors (Figure S4 and Table S1). Interestingly, the hydrophobic quartz interface influenced the hydrogen bonding away from the interface to a greater extent than the hydrophilic, which was found to be comparatively shorter ranged with more defined peaks. Similar long-range perturbation of hydrogen bonding has previously been observed from MD simulations of the water-vapor interface. .53

The first three correlation functions in $\mathrm{h}_{n 0 n}$ show that the hydrophilic surface induces sharper regions of preferential dipole moment orientation, which may reflect hydrogen bonding with the surface groups of the quartz as well as with other interfacial water. The hydrophobic surface, on the other hand, shows more relaxed profiles for all functions, where the $\mathrm{h}_{202}$ function is greatest around the initial peak of the density profile. This reflects a preference for water molecules to align their dipole moments perpendicular to the interface normal (i.e. along the interface). The $\mathrm{h}_{101}$ functions for the hydrophobic system show that at the interface, the water molecules' dipole moments are preferentially orientated towards the bulk region, but at 0.4 to $0.6 \mathrm{~nm}$ away from the surface this preference is reverted to orientate back towards the hydrophobic surface. On the other hand, for the hydrophilic system these same functions are strongly oscillating over the same distance from the direct surface. The higher-order $\mathrm{h}_{n 0 n}$ functions (Figure $\mathrm{S} 5$ ) also show strong oscillations and therefore structure for the hydrophilic system, which is more relaxed for the hydrophobic. 
The length scales over which oscillations in the hydrophobic surface-induced water structure persists are similar to that observed previously for water solvating a planar hydrophobic graphene surface. $\frac{5455}{5}$ For the hydrophobic system, the distribution of angles between water's oxygen and hydrogen bond vector and the interface normal (Figure S6) showed that directly solvating water molecules (within $2.5 \AA$ of the surface) have a tendency to direct one $\mathrm{O}-\mathrm{H}$ vector towards the hydrophobic solid, and the second vector consequently directed slightly inside the water phase, consistent with previous studies of water orientation at hydrophobic surfaces. $\stackrel{56}{58}$ Together, these clarify that both surfaces behave as hydrophilic and hydrophobic, respectively, and as such the aqueous water interacts greater, in terms of both density and molecular orientation, with the hydrophilic quartz surface.

Apart from differences in molecular orientation, the water-water interactions are likely changed due to the perturbation of the usual intermolecular forces in aqueous water due to the presence of each surface. This was investigated by selectively analyzing dipolar interactions between water molecules in proximity to each surface. Following the method of Liu et al., 59 the angular-dependent radial distribution function (RDF), $g^{i j}$, between two dipole moments of a liquid, $\boldsymbol{\mu}_{1}$ of species $i$ and $\boldsymbol{\mu}_{2}$ of species $j$ separated by $\boldsymbol{r}$, is considered in terms of the angular dependent potential of mean force (PMF) as $\underline{59160}$

$$
g^{i j}\left(\boldsymbol{r}, \boldsymbol{\mu}_{1}, \boldsymbol{\mu}_{2}\right)=\exp \left(-\beta \mathcal{W}^{i j}\left(\boldsymbol{r}, \boldsymbol{\mu}_{1}, \boldsymbol{\mu}_{2}\right)\right),
$$

where $\beta=\left(k_{B} T\right)^{-1}, T$ is the absolute temperature, $k_{B}$ is Boltzmann's constant, and $\mathcal{W}^{i j}$ is the PMF. The expansion of the angular-dependent functions in Equation 1 in the basis of rotational invariants, $\frac{61}{{ }_{1}} \Phi_{l_{1} l_{2} l}\left(\hat{\boldsymbol{r}}, \hat{\boldsymbol{\mu}}_{1}, \hat{\boldsymbol{\mu}}_{2}\right)$, gives 


$$
\begin{array}{r}
-\beta \mathcal{W}_{S}^{i j}(r)-\sum_{l_{1}} \sum_{l_{2}} \sum_{l \geq\left|l_{1}-l_{2}\right|}^{l_{1}+l_{2}} \beta \mathcal{W}_{l_{1} l_{2} l}^{i j}(r) \Phi_{1_{1} l_{2} l}= \\
\log \left(g_{S}^{i j}(r)+\sum_{l_{1}} \sum_{l_{2}} \sum_{l \geq\left|l_{1}-l_{2}\right|}^{l_{1}+l_{2}} h_{l_{1} l_{2} l}^{i j}(r) \Phi_{1_{1} l_{2} l}\right),
\end{array}
$$

where $h_{l_{1} l_{2} l}^{i j}$ is an expansion coefficient describing preferential modes of dipole orientations that are selected by $\Phi_{l_{1} l_{2} l}$. The subscript " $S$ " denotes the term that is independent of dipole moment orientation. Gray and Gubbins ${ }^{62}$ describe the allowed values of the indices in the $h_{l_{1} l_{2} l}$ dipolar pair correlation functions. The contribution of dipole correlations $h_{l_{1} l_{2} l}^{i j}$ to the angular averaged PMF, $\omega^{i j}(r)=-k_{B} T \log \left(g_{S}^{i j}(r)\right)$, is expressed as

$$
\begin{aligned}
& \omega^{i j}(r)=\mathcal{W}_{S}^{i j}(r) \\
& -k_{B} T\left\langle\log \left(1+\sum_{l_{1}} \sum_{l_{2}} \sum_{l \geq\left|l_{1}-l_{2}\right|}^{l_{1}+l_{2}} \frac{h_{l_{1} l_{2} l}^{i j}(r)}{g_{S}^{i j}(r)} \Phi_{1_{1} l_{2} l}\right)\right\rangle
\end{aligned}
$$

Expansion of the logarithm in Equation 3 to the second order rotational invariants for correlations between pairs of solvent molecules, i.e. $i=j=s$, yields

$$
\begin{gathered}
\omega^{s s}(r)=\mathcal{W}_{S}^{s s}(r)+\phi(r) \\
+O\left(\left(h_{l_{1} l_{2} l}^{s s}(r)\right)^{3} /\left(g_{S}^{s s}(r)\right)^{3}\right), \\
\phi_{M D}(r)=-k_{B} T \frac{1}{2\left(g_{S}^{s s}(r)\right)^{2}} \sum_{l_{1}} \sum_{l_{2}} \sum_{l \geq\left|l_{1}-l_{2}\right|}^{l_{1}+l_{2}}\left(h_{l_{1} l_{2} l}^{s s}(r)\right)^{2},
\end{gathered}
$$


where $\phi_{M D}(r)$ is an orientation-independent contribution to the PMF arising from orientational correlation between dipoles. Note that $h_{l_{1} l_{2} l}(r)$ can be calculated from MD simulations via $h_{l_{1} l_{2} l}(r)=\langle\Phi\rangle_{\mu 1, \mu 2,|\mathbf{r}|=r}$. We label $\phi_{M D}(r)$ with a subscript "MD", to signify that it is calculated from simulated trajectories. The $\phi_{M D}(r)$ interaction is always negative, so it produces an attractive force between pairs of polar molecules, which has been shown to contribute to the entropy of hydrophobic solvation, $\frac{59}{50}$ to contribute to the long-range structure of polar liquids, including liquid water, $\underline{63}$ to be selectively modulated by different ions,, 64 and to produce a stabilizing force at the water-vapor interface. ${ }^{60}$ It is therefore of interest to compare the strength of this interaction when water molecules are constrained in their orientation by the quartz interface. This is done by calculating the PMF between water molecules when at least one molecule is within a distance $\xi$ from the direct surface, which corresponds to the region in $z$ of the initial peak in $\rho(z)$ from Figure $1 \mathrm{D}$ and H, respectively, averaged over all interactions with every other water molecule in the system.

The water molecules within $\xi$ of the quartz interface exhibit enhanced long-range dipolar interactions with other water molecules (Figure 2). This is particularly visible in the 2nd shell in $\phi_{M D}(r)$ between about 4 and $6 \AA$ of separation between molecules, where the interaction is about 1.8 to 2.8 times stronger than the bulk water-water interaction for both the hydrophilic and hydrophobic surfaces, respectively. This enhancement is likely dominated by interactions with other interfacial water molecules, so we compared $\phi_{M D}(r)$ for when only interfacialbulk water (molecules outside of $\xi$ ) interactions are analyzed, to which the enhancement is less, but still greater than for the bulk water-water interaction. Interestingly, the longerrange behaviour in $\phi_{M D}(r)$ is increased well above the bulk for both quartz systems. In comparing the hydrophobic to hydrophilic systems, it was found that the molecules against the hydrophobic surface experience greater enhancement of $\phi_{M D}(r)$, though the oscillations in the functions are similar between the two systems. These are also similar to what has previously been observed at the liquid water-vapor interface. $\frac{60}{6}$ This points to some generality in the water-water interaction, where the strength differs depending on surface type, but the 


\section{structural features of the interaction remain consistent.}

The $\phi_{M D}(r)$ interactions contribute to the free energy of the liquid, which is written $\operatorname{as}^{59163165}$

$$
G_{\phi}=4 \pi \rho \int_{0}^{\infty} d r r^{2} \phi_{M D}(r) g_{S}(r) .
$$

Following our recent method, $\underline{60}$ the magnitude of this stabilization was calculated from the difference between $\phi_{M D}(r)$ as a function of position in the $z$ dimension, in comparison to bulk $\phi_{M D}(r)$ interactions, $\phi_{M D}^{b}(r)$, such that

$$
\begin{aligned}
\frac{\Delta G_{\phi}\left(z_{i}\right)}{N} & =\frac{1}{2} \int_{0}^{\infty} d r 4 \pi r^{2} \rho g_{S}(r)\left[\phi_{M D}\left(z_{i}, r\right)-\phi_{M D}^{b}(r)\right] \\
& =\frac{1}{2} \int_{0}^{\infty} d r 4 \pi r^{2} \rho g_{S}(r) \Delta \phi\left(z_{i}, r\right) .
\end{aligned}
$$

For these calculations we take $\phi_{M D}^{b}(r)$ from the centre bulk region of each respective system so as to unambiguously obtain the excess quantities at each interfacial region. A comparison of the $\phi_{M D}^{b}(r)$ functions to that obtained from a "true" bulk SPC/E simulation show that the functions match each other well, with the quartz systems being slightly weaker at large separations (Figure S7). A form that is more convenient to the analysis of MD simulations is the equation

$$
\Delta G_{\phi}\left(z_{i}\right)=\frac{1}{2 L^{2}} \sum_{r_{i}} N_{p}\left(z_{i}, r_{i}\right) \Delta \phi\left(z_{i}, r_{i}\right)
$$

where $N_{p}$ is the number of molecules at each $z_{i}$ in separation of $r_{i}$, and $L$ is the length of $x$ and $y$, included so as to normalize by the surface area of the interface.

Equation 9 was evaluated for both quartz systems in Figure 3 (full profile in Figure S8). An increase in the free energy that results from enhanced $\phi_{M D}(r)$ interactions was found as the interfacial region is approached. This was larger in terms of peak height for water 


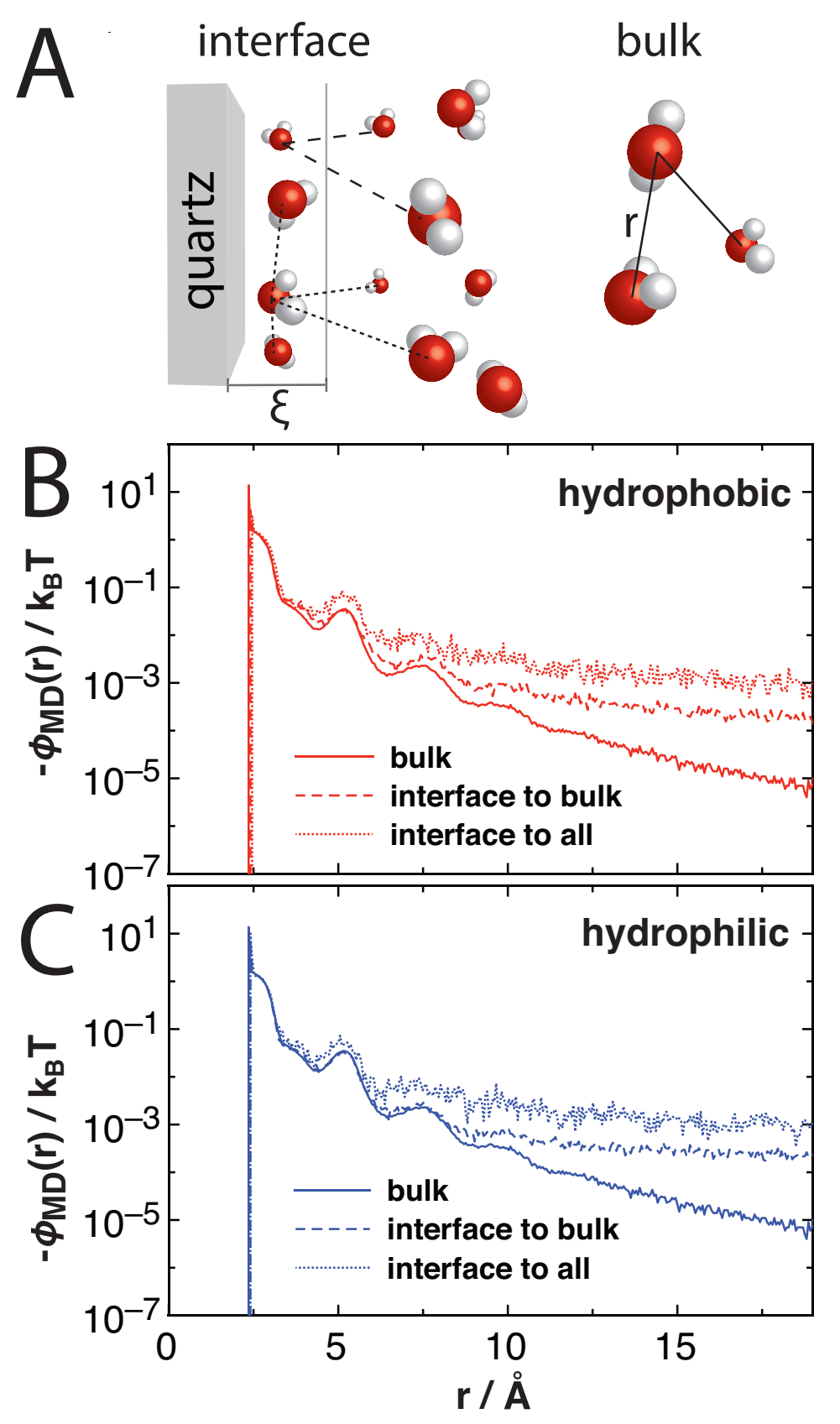

Figure 2. Comparison of the $\phi_{M D}(r)$ interactions between water molecules when one molecule is within the first peak of the surface density profile (from $\mathrm{z}=0$ to $\xi \mathrm{nm}$ ), correlating only with molecules outside of $\xi$ (interface to bulk), or with all molecules including other interfacial molecules (interface to all), along with the bulk interaction (schematic in A), for both the hydrophobic $(\mathrm{B}, \xi=0.55 \mathrm{~nm})$ and hydrophilic $(\mathrm{C}, \xi=0.58 \mathrm{~nm})$ quartz surfaces. 

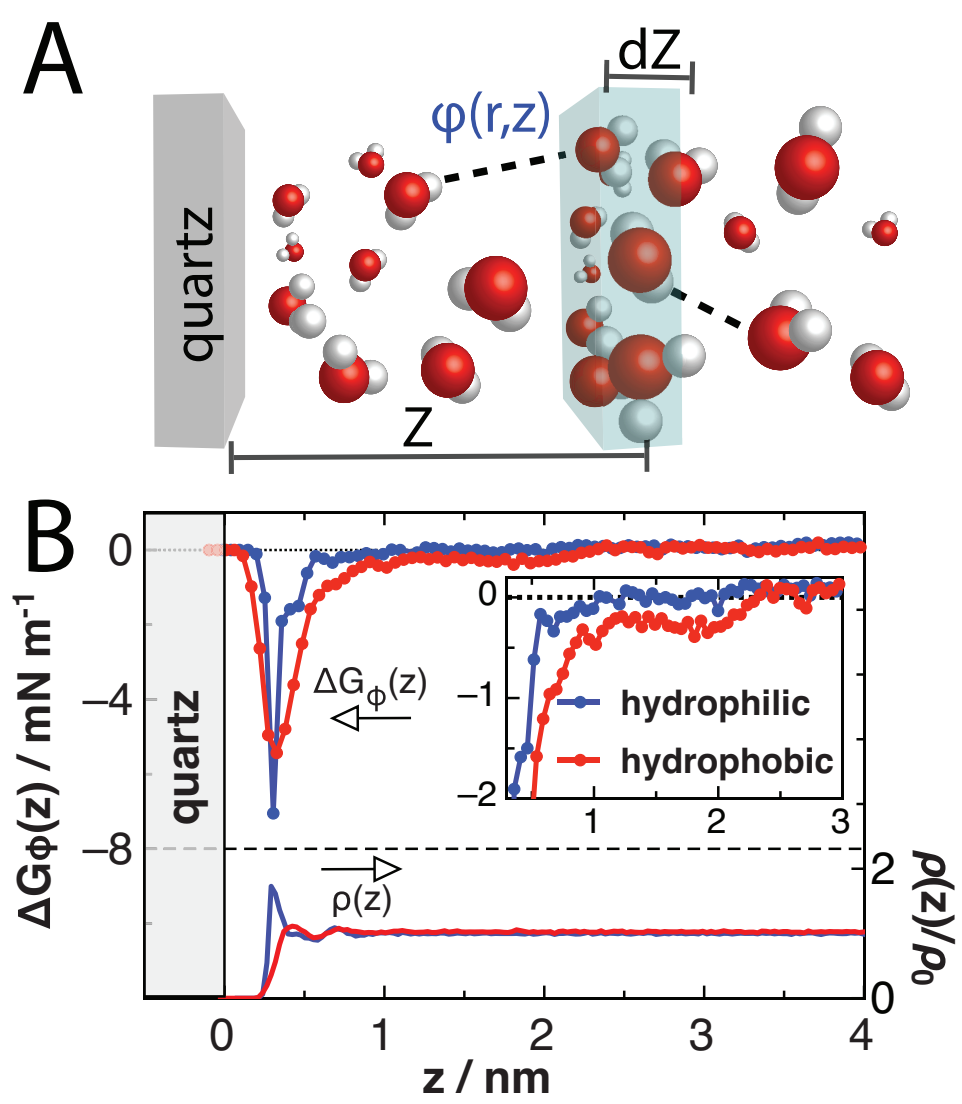

Figure 3. A schematic describing the calculation where the interactions of every water molecule with a "slab" of water at different positions $z$ is computed (A), and the $\Delta G_{\phi}(z)$ functions, as given by Equation 9, for the hydrophilic and hydrophobic quartz systems (B), respectively, along with the corresponding density profiles. 
molecules adjacent to the hydrophilic quartz surface, which is also situated at the first peak of the $\rho(z)$. However, what was also observed was a longer-range stabilization of the water molecules that are adjacent to the hydrophobic quartz surface. The peak height was about $25 \%$ less than that for the hydrophilic surface, though the free energy profile was more extended for the hydrophobic system towards $2.5 \mathrm{~nm}$ of separation from the hard surface. The hydrophilic system only extended for approximately $1 \mathrm{~nm}$ from the hard surface. In other words, Figure 3 shows that there is at least one component of the solvation free energy that is more attractive for hydrophobic quartz than for hydrophilic quartz.

This counter-intuitive result shows the existence of a free energy gradient that water molecules "feel" towards the interfacial region, which results from enhanced orientational correlations between water molecules that interact back towards the bulk phase due to the presence of the hydrophobic interface. This interaction is due to the specific structure of water molecules at the hydrophobic interface, and therefore, at least in part, explains a driving force for that structure (i.e. a mechanism for water molecules to recover free energy due to the presence of the hydrophobic surface). This stabilizing interaction for water molecules towards the interfacial region will likely contribute to the increased molecular density in the interfacial region.

Interestingly, the free energy we find in $\Delta G_{\phi}(z)$ is of the same magnitude of measurable hydrophobic forces at small length scales (for example see reference ${ }^{66}$ ). The attraction in $\Delta G_{\phi}(z)$ at small length scales (below $1 \mathrm{~nm}$ ) is best fitted by a single exponential function of the form

$$
\Delta G_{\phi}(z)=-C \mathrm{e}^{-\mathrm{z} / \mathrm{z}_{0}}
$$

but deviates at larger distances to a more power-like behaviour, fitted by

$$
\Delta G_{\phi}(z)=-\frac{A}{12 \pi z^{2}},
$$


that are both fitted and shown in Figure 4 along with the fitted parameters $C, z_{0}$, and A. This means that the attraction due to $\Delta G_{\phi}$ cannot be adequately captured by a single attractive force law. An important consequence of this result is in relation to the Lifshitz continuum theory of $\mathrm{vdW}$ forces ${ }^{28}$ that is used to rationalize AFM and SFA measurements of hydrophobic forces. The component due to $\phi_{M D}(r)$ is neglected in the assumption of a structureless dielectric continuum, as is the basis in Lifshitz theory, and likely contributes to the inaccuracy of the theory at small separations between surfaces in aqueous water 24 (i.e., there is an attraction of solvent for itself which cannot be calculated through traditional Lifshitz theory).
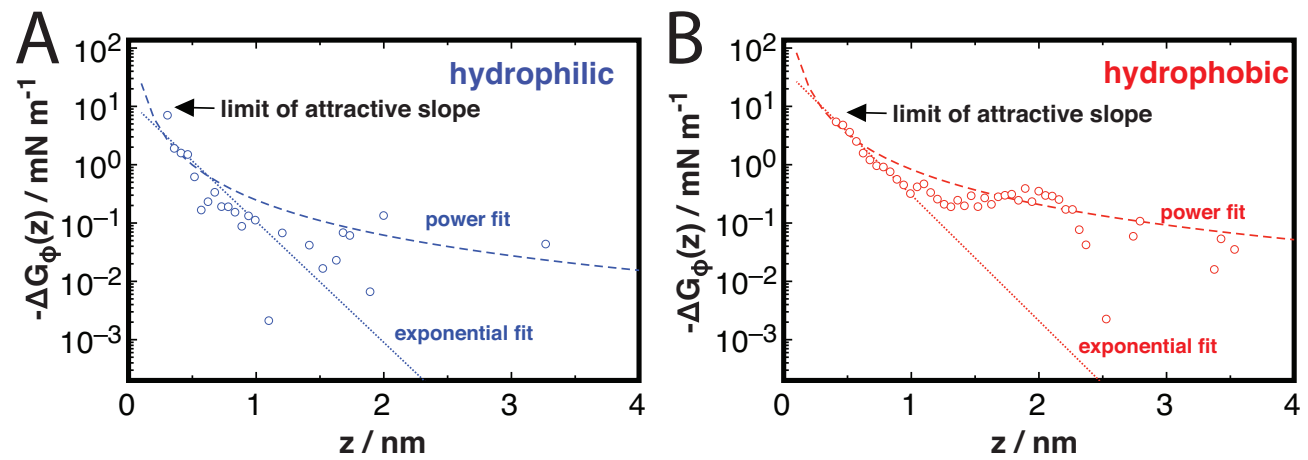

Figure 4. The fitted functions Equation 11 and Equation 10 to the left-hand side attractive slopes in $\Delta G_{\phi}$ of the hydrophilic (A) and hydrophobic (B) quartz systems. The fitted parameters are $A=2.48 \times 10^{-22} \mathrm{~J}, C=12.61 \mathrm{mN} \mathrm{m}^{-1}, z_{0}=0.21 \mathrm{~nm}$, and $A=8.30 \times 10^{-22}$ J, $C=43.71 \mathrm{mN} \mathrm{m}^{-1}, z_{0}=0.20 \mathrm{~nm}$, for the hydrophilic (A) and hydrophobic (B) systems, respectively.

Chun et al. ${ }^{67}$ recently showed how heterogeneity in interfacial solvent structure can result in strengthened short-range dispersion forces between surfaces immersed in aqueous water, over what is predicted within the standard Lifshitz formalization. This is likely linked to the enhanced $\phi_{M D}(r)$ interactions that we have observed for quartz interfacial regions. Additional contributions to the total interfacial forces involve direct vdW attractions with the surface itself, and between two surfaces immersed in water. Furthermore, we point out that our simulated systems do not account for $\mathrm{pH}$ effects at the mineral interface, nor for dissolved gas, which are both omnipresent in experimental measurements of such systems. 
However, our systems have allowed elucidation of an underlying solvation mechanism that in the presence of additional contributions might not be immediately obvious. It is anticipated that the enhanced coupling between dipole moments would become greater towards nanoconfinement regimes ${ }^{68}+70$ and for adsorbed water films on quartz. ${ }^{71}$

Lastly, our results are consistent with experimental analyses for hydrogen bonding and

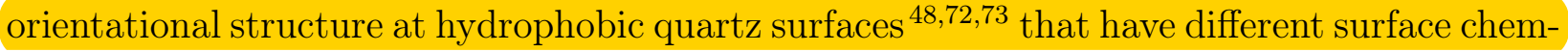
istry to our simulated systems. Moreover, similar enhancement of the $\phi_{M D}(r)$ interactions have previously been observed ${ }^{60}$ at the water liquid-vapor interface. Therefore, we expect similar enhancement of water-water attractive interactions towards other hydrophobic interfaces, including "soft" water-oil interfaces where the instantaneous fluctuations in structure differ considerably from "hard" interfaces. The free energy $\Delta G_{\phi}$ will also depend on surface geometry, whereby the dielectric profiles can differ markedly between planar and curved geometries, along with the long-range electrostatic ion-ion interactions. ${ }^{7475}$

\section{Conclusions}

In summary, we have provided a method for calculating dipolar forces directly from simulation of liquids that are solvating hard quartz surfaces. The contribution of these dipolar interactions to the free energy of the fluid, as a function of distance from the surface, has revealed a gradient increasing in attraction towards the interface that results from changed molecular orientations of the direct solvating water molecules. This was found to exert a longer-ranged stabilization, and hence attraction of water molecules towards a model hydrophobic quartz interface, than to a model hydrophilic interface. These studies have therefore revealed an additional component of the solvation of hydrophobic surfaces by aqueous water, ultimately adding to our understanding of hydrophobicity. 


\section{Associated Content}

Supporting Information. Theoretical details on contact angles, ion density, hydrogen bonding, full $h_{n 0 n}(z)$ functions, bulk system comparisons, and $\Delta G_{\phi}(z)$ functions.

\section{Author Information}

\section{Corresponding Authors}

*E-mail: besford@ipfdd.de

*E-mail: andrew.christofferson@rmit.edu.au

\section{ORCID}

Quinn A. Besford: 0000-0002-1779-9176

Andrew J. Christofferson: 0000-0003-0904-6630

Jens-Uwe Sommer: 0000-0001-8239-3570

Richard H. Henchman: 0000-0002-0461-6625

\section{Notes}

The authors declare no competing financial interest.

\section{Acknowledgments}

We gratefully acknowledge Prof. Andreas Fery, Prof. Chris McConville, Dr. Maximilian Seuss, Dr. Günter Auernhammer, Dr. Maoyuan Liu, and Dr. Matthew Penna for stimulating discussions, and the Spartan HPC system at The University of Melbourne for allocation of computational time (punim0578). Part of this research was funded by the Alexander von Humboldt foundation (Q.A.B.), and the Engineering and Physical Sciences Research Council (EPSRC) under grant codes EP/L015218/1 and EP/N025105/1 (J.K.). 


\section{References}

(1) Vogler, E. A. Structure and Reactivity of Water at Biomaterial Surfaces. Adv. Colloid Interface Sci. 1998, 74, 69-117.

(2) Liang, Y.; Tsuji, S.; Jia, J.; Tsuji, T.; Matsuoka, T. Modeling CO2-Water-Mineral Wettability and Mineralization for Carbon Geosequestration. Acc. Chem. Res. 2017, $50,1530-1540$.

(3) Zhang, X.; Sewell, T. E.; Glatz, B.; Sarupria, S.; Getman, R. B. On the Water Structure at Hydrophobic Interfaces and the Roles of Water on Transition-metal Catalyzed Reactions: A Short Review. Catal. Today 2017, 285, 57-64.

(4) Ducker, W. A.; Mastropietro, D. Forces Between Extended Hydrophobic Solids: Is There a Long-range Hydrophobic Force? Curr. Opin. Colloid Interface Sci. 2016, 22, $51-58$.

(5) Ninham, B. W.; Lo Nostro, P. Molecular Forces and Self Assembly in Colloid, Nano Sciences and Biology; Cambridge University Press: Cambridge, 2010.

(6) Lo Nostro, P.; Ninham, B. W. After DLVO: Hans Lyklema and the Keepers of the Faith. Adv. Colloid Interface Sci. 2020, 276, 102082.

(7) Doshi, D. A.; Watkins, E. B.; Israelachvili, J. N.; Majewski, J. Reduced Water Density at Hydrophobic Surfaces: Effect of Dissolved Gases. Proc. Natl. Acad. Sci. U. S. A. 2005, 102, 9458-9462.

(8) Khatib, R.; Backus, E. H.; Bonn, M.; Perez-Haro, M. J.; Gaigeot, M. P.; Sulpizi, M. Water Orientation and Hydrogen-bond Structure at the Fluorite/Water Interface. Scientific Reports 2016, 6, 1-10.

(9) Zhu, Y.; Granick, S. Viscosity of Interfacial Water. Phys. Rev. Lett. 2001, 87, 1-4. 
(10) Fumagalli, L.; Esfandiar, A.; Fabregas, R.; Hu, S.; Ares, P.; Janardanan, A.; Yang, Q.; Radha, B.; Taniguchi, T.; Watanabe, K.; et al., Anomalously Low Dielectric Constant of Confined Water. Science 2018, 360, 1339-1342.

(11) Brown, M. A.; Goel, A.; Abbas, Z. Effect of Electrolyte Concentration on the Stern Layer Thickness at a Charged Interface. Angew. Chem. Int. Ed. 2016, 55, 3790-3794.

(12) Peng, J.; Guo, J.; Hapala, P.; Cao, D.; Ma, R.; Cheng, B.; Xu, L.; Ondráček, M.; Jelínek, P.; Wang, E.; Jiang, Y. Weakly Perturbative Imaging of Interfacial Water with Submolecular Resolution by Atomic Force Microscopy. Nat. Chem. 2018, 9, 1-7.

(13) Von Rudorff, G. F.; Jakobsen, R.; Rosso, K. M.; Blumberger, J. Fast Interconversion of Hydrogen Bonding at the Hematite (001)-Liquid Water Interface. J. Phys. Chem. 2016, 7, 1155-1160.

(14) Godawat, R.; Jamadagni, S. N.; Garde, S. Characterizing Hydrophobicity of Interfaces by Using Cavity Formation, Solute Binding, and Water Correlations. Proc. Natl. Acad. Sci. U.S.A. 2009, 106, 15119-15124.

(15) Patel, A. J.; Varilly, P.; Jamadagni, S. N.; Acharya, H.; Garde, S.; Chandler, D. Extended Surfaces Modulate Hydrophobic Interactions of Neighboring Solutes. Proc. Natl. Acad. Sci. U.S.A. 2011, 108, 17678-17683.

(16) Sato, T.; Sasaki, T.; Ohnuki, J.; Umezawa, K.; Takano, M. Hydrophobic Surface Enhances Electrostatic Interaction in Water. Phys. Rev. Lett. 2018, 121, 206002.

(17) Pfeiffer-Laplaud, M.; Gaigeot, M. P. Adsorption of Singly Charged Ions at the Hydroxylated (0001) $\alpha$-Quartz/Water Interface. J. Phys. Chem. C 2016, 120, 4866-4880.

(18) Björneholm, O.; Hansen, M. H.; Hodgson, A.; Liu, L. M.; Limmer, D. T.; Michaelides, A.; Pedevilla, P.; Rossmeisl, J.; Shen, H.; Tocci, G.; Tyrode, E.; 
Walz, M. M.; Werner, J.; Bluhm, H. Water at Interfaces. Chem. Rev. 2016, 116, 76987726.

(19) Ohno, P. E.; Wang, H. F.; Geiger, F. M. Second-order Spectral Lineshapes from Charged Interfaces. Nat. Comm. 2017, 8, 1-9.

(20) Ostroverkhov, V.; Waychunas, G.; Shen, Y. New Information on Water Interfacial Structure Revealed by Phase-Sensitive Surface Spectroscopy. Phys. Rev. Lett. 2005, 94, 046102.

(21) Du, Q.; Freysz, E.; Shen, Y. R. Vibrational Spectra of Water Molecules at Quartz/Water Interfaces. Phys. Rev. Lett. 1994, 72, 238-241.

(22) Smirnov, K. S. Structure and Sum-frequency Generation Spectra of Water on Uncharged Q4 Silica Surfaces: a Molecular Dynamics Study . Phys. Chem. Chem. Phys. 2020, 2033-2045.

(23) Smith, A. M.; Borkovec, M.; Trefalt, G. Forces Between Solid Surfaces in Aqueous Electrolyte Solutions. Adv. Colloid Interface Sci. 2020, 275, 102078.

(24) Meyer, E. E.; Rosenberg, K. J.; Israelachvili, J. Recent Progress in Understanding Hydrophobic Interactions. Proc. Natl. Acad. Sci. U.S.A. 2006, 103, 15739-46.

(25) Tabor, R. F.; Grieser, F.; Dagastine, R. R.; Chan, D. Y. The hydrophobic force: Measurements and methods. Phys. Chem. Chem. Phys. 2014, 16, 18065-18075.

(26) Meyer, E. E.; Lin, Q.; Hassenkam, T.; Oroudjev, E.; Israelachvili, J. N. Origin of the Long-range Attraction Between Surfactant-coated Surfaces. Proc. Natl. Acad. Sci. U.S.A. 2005, 102, 6839-6842.

(27) Attard, P. Bridging Bubbles between Hydrophobic Surfaces. Langmuir 1996, 12, 16931695. 
(28) Lifshitz, E. The Theory of Molecular Attractive Forces Between Solids. Soviet Physics JETP 1956, 2, 73-83.

(29) Covert, P. A.; Hore, D. K. Geochemical Insight from Nonlinear Optical Studies of Mineral-Water Interfaces. Annu. Rev. Phys. Chem. 2016, 67, 233-257.

(30) Ohno, P. E.; Saslow, S. A.; Wang, H. F.; Geiger, F. M.; Eisenthal, K. B. Phasereferenced Nonlinear Spectroscopy of the $\alpha$-Quartz/Water Interface. Nat. Comm. 2016, $7,1-5$.

(31) Lv, Y.; Wang, X.; Yu, X.; Zheng, S.; Wang, S.; Zhang, Y.; Du, H. Adsorption Behaviors and Vibrational Spectra of Hydrogen Peroxide Molecules at Quartz/Water Interfaces. Phys. Chem. Chem. Phys. 2017, 19, 7054-7061.

(32) Wang, C.; Cao, X. L.; Guo, L. L.; Xu, Z. C.; Zhang, L.; Gong, Q. T.; Zhang, L.; Zhao, S. Effect of Adsorption of Catanionic Surfactant Mixtures on Wettability of Quartz Surface. Colloid. Surface. A. 2016, 509, 564-573.

(33) Deng, Y.; Xu, L.; Lu, H.; Wang, H.; Shi, Y. Direct Measurement of the Contact Angle of Water Droplet on Quartz in a Reservoir Rock with Atomic Force Microscopy. Chem. Eng. Sci. 2018, 177, 445-454.

(34) Liu, C.; Min, F.; Liu, L.; Chen, J. Density Functional Theory Study of Water Molecule Adsorption on the $\alpha$-Quartz (001) Surface with and without the Presence of $\mathrm{Na}+$, $\mathrm{Mg} 2+$, and $\mathrm{Ca} 2+$. ACS Omega 2019, 4, 12711-12718.

(35) Jia, J.; Liang, Y.; Tsuji, T.; Miranda, C. R.; Masuda, Y.; Matsuoka, T. Ab Initio Molecular Dynamics Study of Carbonation and Hydrolysis Reactions on Cleaved Quartz (001) Surface. J. Phys. Chem. C 2019, 123, 4938-4948.

(36) Wander, M. C.; Clark, A. E. Structural and Dielectric Properties of Quartz-water Interfaces. J. Phys. Chem. C 2008, 112, 19986-19994. 
(37) Parashar, S.; Lesnicki, D.; Sulpizi, M. Increased Acid Dissociation at the Quartz/Water Interface. J. Phys. Chem. 2018, 9, 2186-2189.

(38) Gor, G. Y.; Bernstein, N. Adsorption-Induced Surface Stresses of the Water/Quartz Interface: Ab Initio Molecular Dynamics Study. Langmuir 2016, 32, 5259-5266.

(39) Abramov, A.; Keshavarz, A.; Iglauer, S. Wettability of Fully Hydroxylated and Alkylated (001) $\alpha$-Quartz Surface in Carbon Dioxide Atmosphere. J. Phys. Chem. C 2019, 123, 9027-9040.

(40) DelloStritto, M. J.; Kubicki, J. D.; Sofo, J. O. Effect of Ions on H-Bond Structure and Dynamics at the Quartz(101)-Water Interface. Langmuir 2016, 32, 11353-11365.

(41) Dauber-Osguthorpe, P.; Roberts, V.; Osguthorpe, D.; Wolff, J.; Genest, M.; Hagler, A. Structure and Energetics of Ligand Binding to Proteins : Escherichia coZi Dihydrofolate Reductase-Trimethoprim, A Drug-Receptor System. Proteins 1988, 47, 31-47.

(42) Berendsen, H. J.; Grigera, J. R.; Straatsma, T. P. The Missing Term in Effective Pair Potentials. J. Phys. Chem. 1987, 91, 6269-6271.

(43) Weerasinghe, S.; Smith, P. E. A Kirkwood-Buff Derived Force Field for Sodium Chloride in Water. J. Chem. Phys. 2003, 119, 11342-11349.

(44) Plimpton, S. Fast Parallel Algorithms for Short-Range Molecular Dynamics. J. Comput. Phys. 1995, 117, 1-19.

(45) Hockney, R. W.; Eastwood, J. W. Computer Simulation Using Particles; Taylor and Francis Group: New York, 1988.

(46) Wright, L. B.; Freeman, C. L.; Walsh, T. R. Benzene Adsorption at the Aqueous (0 11 1) $\alpha$-Quartz Interface: Is Surface Flexibility Important? Mol. Simul. 2013, 39, 1093-1102. 
(47) Emami, F. S.; Puddu, V.; Berry, R. J.; Varshney, V.; Patwardhan, S. V.; Perry, C. C.; Heinz, H. Force Field and a Surface Model Database for Silica to Simulate Interfacial Properties in Atomic Resolution. Chem. Mater. 2014, 26, 2647-2658.

(48) Asanuma, H.; Noguchi, H.; Uosaki, K.; Yu, H.-Z. Water Structure at Superhydrophobic Quartz/Water Interfaces: A Vibrational Sum Frequency Generation Spectroscopy Study. J. Phys. Chem. C 2009, 113, 21155-21161.

(49) Jungwirth, P.; Laage, D. Ion-Induced Long-Range Orientational Correlations in Water: Strong or Weak, Physiologically Relevant or Unimportant, and Unique to Water or Not? J. Phys. Chem. Lett. 2018, 9, 2056-2057.

(50) Chu, M.; Miller, M.; Dutta, P. Interfacial Density Profiles of Polar and Nonpolar Liquids at Hydrophobic Surfaces. Langmuir 2020, 36, 906-910.

(51) Mezger, M.; Reichert, H.; Schöder, S.; Okasinski, J.; Schöder, H.; Dosch, H.; Palms, D.; Ralston, J.; Honkimäki, V. High-resolution In Situ X-ray Study of the Hydrophobic Gap at the Water-octadecyl-trichlorosilane Interface. Proc. Natl. Acad. Sci. U.S.A. 2006, 103, 18401-18404.

(52) Irudayam, S. J.; Henchman, R. H. Prediction and Interpretation of the Hydration Entropies of Monovalent Cations and Anions. Mol. Phys. 2011, 109, 37-48.

(53) Irudayam, S. J.; Henchman, R. H. Long-range Hydrogen-bond Structure in Aqueous Solutions and the Vapor-water Interface. J. Chem. Phys. 2012, 137, 034508.

(54) Malaspina, D.; Schulz, E.; Alarcón, L.; Frechero, M.; Appignanesi, G. Structural and Dynamical Aspects of Water in Contact with a Hydrophobic Surface. Eur. Phys. J. E 2010, 32, 35-42.

(55) Alarcón, L.; Malaspina, D.; Schulz, E.; Frechero, M.; Appignanesi, G. Structure and 
Orientation of Water Molecules at Model Hydrophobic Surfaces with Curvature: From Graphene Sheets to Carbon Nanotubes and Fullerenes. Chem. Phys. 2011, 388, 47-56.

(56) Janeček, J.; Netz, R. R. Interfacial Water at Hydrophobic and Hydrophilic Surfaces: Depletion Versus Adsorption. Langmuir 2007, 23, 8417-8429.

(57) Strazdaite, S.; Versluis, J.; Bakker, H. J. Water Orientation at Hydrophobic Interfaces. J. Chem. Phys. 2015, 143, 084708.

(58) Giovambattista, N.; Debenedetti, P. G.; Rossky, P. J. Effect of Surface Polarity on Water Contact Angle and Interfacial Hydration Structure. J. Phys. Chem. B 2007, 111, 9581-9587.

(59) Liu, M.; Besford, Q. A.; Mulvaney, T.; Gray-Weale, A. Order and Correlation Contributions to the Entropy of Hydrophobic Solvation. J. Chem. Phys. 2015, 142, 114117$1-114117-15$.

(60) Besford, Q. A.; Liu, M.; Christofferson, A. J. Stabilizing Dipolar Interactions Drive Specific Molecular Structure at the Water Liquid-Vapor Interface. J. Phys. Chem. B 2018, 122, 8309-8314.

(61) Hansen, J.-P.; McDonald, I. R. Theory of Simple Liquids, 2nd ed.; Academic Press: London, 1986.

(62) Gray, C.; Gubbins, K. Theory of Molecular Fluids; Oxford Univeristy Press: New York, 1984.

(63) Besford, Q. A.; Christofferson, A. J.; Liu, M.; Yarovsky, I. Long-Range Dipolar Order and Dispersion Forces in Polar Liquids. J. Chem. Phys. 2017, 147, 194503.

(64) Besford, Q.; Liu, M.; Gray-Weale, A. Pair Correlations that Link the Hydrophobic and Hofmeister Effects. Phys. Chem. Chem. Phys. 2016, 18, 14949-14959. 
(65) Hansen, J. P.; McDonald, I. R. Theory of Simple Liquids, 2nd ed.; Academic Press: London, 2002.

(66) Hammer, M.; Anderson, T.; Chaimovich, A.; Shell, M.; Israelachvili, J. The Search for the Hydrophobic Force Law. Farad. Discuss. 2010, 146, 299-308.

(67) Chun, J.; Mundy, C. J.; Schenter, G. K. The Role of Solvent Heterogeneity in Determining the Dispersion Interaction Between Nanoassemblies. J. Phys. Chem. B 2015, 119, 5873-5881.

(68) Diao, Y.; Espinosa-Marzal, R. M. Molecular Insight into the Nanoconfined Calcitesolution Interface. Proc. Natl. Acad. Sci. U.S.A. 2016, 113, 12047-12052.

(69) Giovambattista, N.; Rossky, P. J.; Debenedetti, P. G. Effect of Pressure on the Phase Behavior and Structure of Water Confined between Nanoscale Hydrophobic and Hydrophilic Plates. Phys. Rev. E 2006, 73, 041604.

(70) Li, J.; Cao, W.; Li, J.; Ma, M.; Luo, J. Molecular Origin of Superlubricity between Graphene and a Highly Hydrophobic Surface in Water. J. Phys. Chem. Lett. 2019, 10, $2978-2984$.

(71) Pashley, R.; Kitchener, J. Surface Forces in Adsorbed Multilayers of Water on Quartz. J. Colloid Interface Sci. 1979, 71, 491-500.

(72) Ye, S.; Nihonyanagi, S.; Uosaki, K. Sum Frequency Generation (SFG) Study of the pH-dependent Water Structure on a Fused Quartz Surface Modified by an Octadecyltrichlorosilane (OTS) Monolayer. Phys. Chem. Chem. Phys. 2001, 3, 3463-3469.

(73) Du, Q.; Freysz, E.; Shen, R. Surface Vibrational Spectroscopic Studies of Hydrogen Bonding and Hydrophobicity. Science 1994, 264, 826-828. 
(74) Loche, P.; Ayaz, C.; Schlaich, A.; Uematsu, Y.; Netz, R. Giant Axial Dielectric Response in Water-Filled Nanotubes and Effective Electrostatic Ion-Ion Interactions from a Tensorial Dielectric Model. J. Phys. Chem. B 2019, 123, 10850-10857.

(75) Loche, P.; Ayaz, C.; Wolde-Kidan, A.; Schlaich, A.; Netz, R. Universal and Nonuniversal Aspects of Electrostatics in Aqueous Nanoconfinement. J. Phys. Chem. B 2020, 124, 4365-4371. 
TOC Graphic

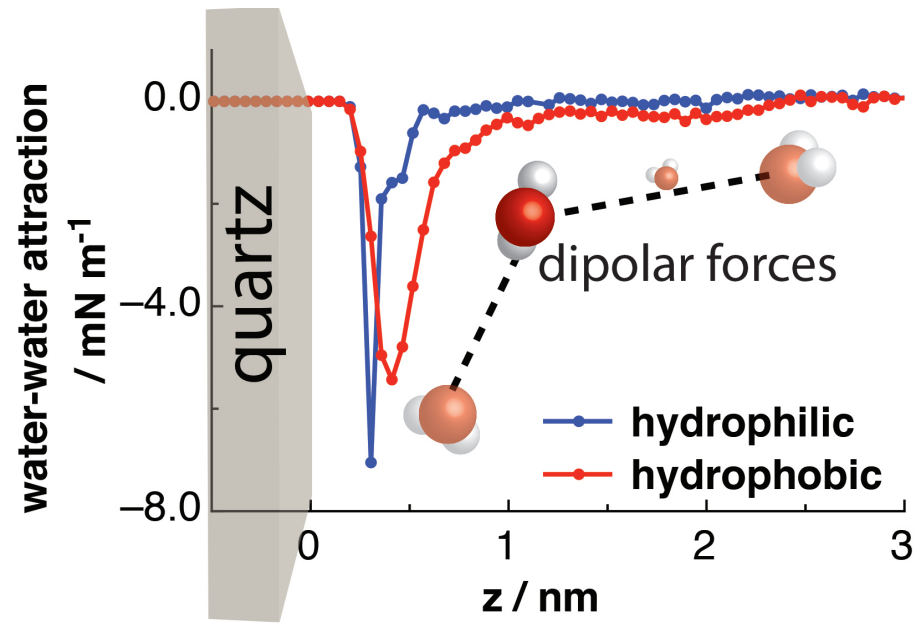

